\title{
Allele-specific programming of Npy and epigenetic effects of physical activity in a genetic model of depression
}

\author{
PA Melas ${ }^{1,2}$, A Lennartsson ${ }^{3}$, H Vakifahmetoglu-Norberg ${ }^{4}$, Y Wei $^{1,2}$, E Åberg $^{5}$, M Werme $^{6}$, M Rogdaki $^{1,2}$, M Mannervik $^{4}$, G Wegener $^{7,8}$, \\ S Brené ${ }^{5}$, AA Mathé ${ }^{6}$ and $C$ Lavebratt ${ }^{1,2}$
}

Neuropeptide Y (NPY) has been implicated in depression, emotional processing and stress response. Part of this evidence originates from human single-nucleotide polymorphism (SNP) studies. In the present study, we report that a SNP in the rat Npy promoter (C/T; rs105431668) affects in vitro transcription and DNA-protein interactions. Genotyping studies showed that the C-allele of rs 105431668 is present in a genetic rat model of depression (Flinders sensitive line; FSL), while the SNP's T-allele is present in its controls (Flinders resistant line; FRL). In vivo experiments revealed binding of a transcription factor (CREB2) and a histone acetyltransferase (Ep300) only at the SNP locus of the FRL. Accordingly, the FRL had increased hippocampal levels of Npy mRNA and H3K18 acetylation; a gene-activating histone modification maintained by Ep300. Next, based on previous studies showing antidepressant-like effects of physical activity in the FSL, we hypothesized that physical activity may affect Npy's epigenetic status. In line with this assumption, physical activity was associated with increased levels of Npy mRNA and H3K18 acetylation. Physical activity was also associated with reduced mRNA levels of a histone deacetylase (Hdac5). Conclusively, the rat rs 105431668 appears to be a functional Npy SNP that may underlie depression-like characteristics. In addition, the achieved epigenetic reprogramming of $\mathrm{Npy}$ provides molecular support for the putative effectiveness of physical activity as a non-pharmacological antidepressant.

Translational Psychiatry (2013) 3, e255; doi:10.1038/tp.2013.31; published online 7 May 2013

\section{Introduction}

One of the few candidate genes showing reproducibility among genetic association studies of depression is neuropeptide Y (NPY). ${ }^{1-5}$ NPY is widely expressed in the central nervous system and has been suggested to act as an anxiolytic peptide that confers mental resilience ${ }^{6-9}$ through activation of limbic NPY-Y1 receptors. ${ }^{10}$ In addition, NPY was found to regulate neurogenesis in the hippocampus; ${ }^{11}$ a brain region appearing dysfunctional and with reduced volumes in severely depressed patients. ${ }^{12-16}$ The human NPY harbors functional single-nucleotide polymorphisms (SNPs) that control NPYs transcriptional activity. ${ }^{17}$ One of these SNPs (rs16147: C/T) is known to affect emotional processing ${ }^{3,17,18}$ and its effect is modulated by early-life adversities. ${ }^{5,19-21}$ However, the downstream molecular consequences of functional NPY SNPs, like rs16147, remain unknown and in vivo experimental research has been lacking due to the absence of suitable genetic models.

The amino-acid sequence of the mature human NPY is identical to the rat and mouse sequences, and all three species have conserved NPY promoter sequence elements, including the TATA- and GC-boxes. ${ }^{22}$ This makes rodents a suitable model for translational gene-regulatory research of Npy. A Sprague-Dawley rat model that displays a genetic susceptibility to depressive behavior is the Flinders sensitive line (FSL) which, compared with its controls (Flinders resistant line; FRL), shows changes consistent with the NPYergic hypothesis of depression. ${ }^{23-27}$ Apart from depression-like behavioral characteristics like psychomotor retardation and sleep disturbances, ${ }^{28}$ the FSL have reduced hippocampal volumes, ${ }^{29}$ show a dysfunctional regulation of glutamate transmission ${ }^{30}$ and exhibit emotional memory impairments. ${ }^{31}$ Additionally, physical activity in the form of wheel running has been shown to increase cell proliferation in the hippocampus of the FSL and to have antidepressant-like effects. ${ }^{32,33}$ However, the genetic components underlying the observed depression-like phenotypes and molecular characteristics of this genetic model have never been explored.

In this report, we hypothesized that hippocampal dysregulation of Npy in the FSL may be caused by functional SNPs in

\footnotetext{
${ }^{1}$ Department of Molecular Medicine and Surgery, Neurogenetics Unit, Karolinska Institutet, Karolinska University Hospital, Stockholm, Sweden; ${ }^{2}$ Center for Molecular Medicine, Karolinska University Hospital, Stockholm, Sweden; ${ }^{3}$ Department of Biosciences and Nutrition, Center for Biosciences, Karolinska Institutet, Stockholm, Sweden; ${ }^{4}$ Department of Developmental Biology, Wenner-Gren Institute, Arrhenius Laboratories, Stockholm University, Stockholm, Sweden; ${ }^{5}$ Department of Neurobiology, Care Sciences and Society, Karolinska University Hospital Huddinge, Stockholm, Sweden; ${ }^{6}$ Department of Clinical Neuroscience, Section for Psychiatry, Karolinska Institutet, Stockholm, Sweden; ${ }^{7}$ Centre for Psychiatric Research, Aarhus University Hospital, Risskov, Denmark and ${ }^{8}$ Unit for Drug Research and Development, School of Pharmacy (Pharmacology), North-West University, Potchefstroom, South Africa

Correspondence: Dr PA Melas, Department of Molecular Medicine and Surgery, Center for Molecular Medicine, Neurogenetics Unit, Karolinska Institutet, Karolinska University Hospital, CMM L8:00, 17176 Stockholm, Sweden.

E-mail: philippe.melas@ki.se
}

Keywords: Creb; Ep300; hippocampus; histone modifications; mood disorders; neuropeptide Y

Received 16 October 2012; revised 19 February 2013; accepted 15 March 2013 
the promoter region, similar to rs16147 in humans. Accordingly, we found a functional SNP (C/T; rs105431668) located in Npy's core promoter, between the gene's TATA-box and the transcription start site (TSS). In vitro and in vivo experiments demonstrated that this SNP affected gene expression levels and was responsible for DNA-protein interactions which, in turn, were associated with distinct epigenetic states of Npy. Additionally, the latter epigenetic states were found to be affected by physical activity, providing molecular evidence for the putative effectiveness of physical activity as a nonpharmacological antidepressant.

\section{Materials and methods}

Tissue samples. Tissue samples included hippocampi from adult FRL and FSL rats that were dissected and immediately stored at $-70^{\circ} \mathrm{C}$ until subsequent experimental analyses. The number of samples used for each experiment is given in the corresponding methodological section. All experiments were approved by the Ethical Committee for protection of animals at the Karolinska Institutet.

DNA/RNA extraction and reverse transcription. DNA was isolated using the QIAamp DNA Mini Kit (Qiagen $\mathrm{GmbH}$, Hilden, Germany) or a standard isopropanol-based extraction protocol. Total RNA was isolated using the RNeasy Lipid Tissue Mini Kit (Qiagen) and was treated with DNase I (Qiagen) to eliminate contaminating DNA. The RNA quality was examined on a $1 \%$ agarose gel and total DNA/RNA concentrations were determined spectrophotometrically using the NanoDrop ND-1000 Spectrophotometer (NanoDrop Technologies Inc., Wilmington, DE, USA). Complementary DNA was synthesized by reverse transcription of total RNA using SuperScript III First-Strand Synthesis System for real-time PCR (RT-PCR) (Invitrogen, Carlsbad, CA, USA). DNA/complementary DNA was stored at $-20^{\circ} \mathrm{C}$ and RNA at $-70^{\circ} \mathrm{C}$.

DNA sequencing. DNA sequencing was performed to examine genetic differences between FRL and FSL. For this reason, the proximal upstream promoter and all coding exons of Npy were sequenced in FRL $(n=6)$ and FSL $(n=6)$. Genomic DNA was used for sequencing of the proximal promoter, which was restricted to 768 nucleotides (nts) upstream from the TSS $(-768$ to +1$)$, and complementary DNA was used for sequencing of the amino-acid-coding regions. Sequencing reactions were performed bidirectionally on an ABI 3730 DNA Analyzer (Applied Biosystems Inc., Foster City, CA, USA), using the BigDye Terminator v.3.1 Cycle Sequencing kit (Applied Biosystems). The sequencing primers used are given in Supplementary Materials/Methods. Genetic variation was assessed using the Geneious software version 4.8 (Biomatters Ltd, Auckland, New Zealand ${ }^{34}$ ).

SNP genotyping by pyrosequencing. The three promoter SNPs identified in the initial genetic screening by DNA sequencing (rs8153612 (A/G), rs107377172 (A/C), rs105431668 (C/T)) were subsequently genotyped in a larger separate set of FRL $(n=19)$ and FSL $(n=24)$ using pyrosequencing methodology. Pyrosequencing was performed on a PSQ 96 MA platform according to the manufacturer's protocol (Qiagen). The primer pairs used for genotyping are given in Supplementary Materials/Methods.

Gene expression by quantitative RT-PCR. Quantitative RT-PCR (qRT-PCR) was performed to measure mRNA levels of candidate genes using whole hippocampal tissue. First, Npy mRNA levels were measured in naive FRL and FSL rats ( $n=8 \mathrm{FRL}$ and $n=10 \mathrm{FSL}$ ). We have previously shown that the rat Npy mRNA exists in two splice variants; a 'long' protein-coding variant and a 'short' putatively noncoding variant. ${ }^{35}$ All Npy mRNA experiments performed in this study were designed to detect only the coding variant. Following wheel running experiments (see section below), both Npy mRNA levels ( $n=8$ FSL, $n=7$ FSL-runners; outliers: $n=2 \mathrm{FSL}$ and $n=1 \mathrm{FSL}-$ runner) and mRNA levels of candidate histone modifying enzymes (histone acetyltransferase Ep300, and nuclear located class I, II, III and IV histone deacetylases (HDACs); $n=6$ FSL, $n=6$ FSL-runners; outliers: $n=1 \mathrm{FSL}$ and $n=1 \mathrm{FSL}-$ runner) were measured. TaqMan Gene Expression Assays (Applied Biosystems) or Power SYBR Green (Applied Biosystems) was used for qRTPCR experiments. Amplifications of target genes and housekeeping gene were performed in triplicates using complementary DNA. Gene amplifications were performed on an $\mathrm{ABI}$ PRISM 7900 HT Sequence Detection System (Applied Biosystems) and data were obtained as threshold cycle (Ct) values. Relative quantifications of gene expression were calculated using the comparative Ct method: $2^{-\Delta \Delta \mathrm{Ct}}$, where $\Delta \mathrm{Ct}=\mathrm{Ct}$ Target-Ct Housekeeping gene, and $\Delta \Delta \mathrm{Ct}=\Delta \mathrm{Ct}$ Target $-\Delta \mathrm{Ct}$ Calibrator. The panel of tested genes and their corresponding primer pairs, together with thermal cycling conditions, are shown in Supplementary Table S1. Only one gene ( $\mathrm{Hdac} 7$ ) yielded Ct values $\geqslant 35$ and was, therefore, not analyzed.

Gene expression of $\mathrm{Npy}$ by in situ hybridization. To measure Npy mRNA levels in distinct hippocampal regions (cornu ammonis 1 (CA1), cornu ammonis 3 (CA3) and dentate gyrus), in situ hybridization experiments were carried out in FRL $(n=9)$ and FSL $(n=6)$. The hybridization protocol used was as previously described. ${ }^{36}$

Luciferase reporter assay. A luciferase reporter assay was used to investigate the putative functionality of the three SNPs located in the Npy promoter region of FRL/FSL. The upstream Npy promoter region (containing all the three SNPs: 5'- rs8153612-rs107377172-rs105431668-3') and the core promoter region (containing only rs105431668; located between the gene's TATA-box and the TSS), were individually cloned into the Kpnl and Bglll sites of a pGL3 Basic Luciferase Reporter Vector (Promega Corp., Fitchburg, WI, USA). The empty pGL3 Basic Luciferase Reporter vector without any insert and a control-Luciferase vector with the SV40 enhancer and promoter were used as negative and positive controls, respectively. A Renilla luciferase vector driven by a Pol III promoter was used to control for transfection efficiency. Construct transfections were performed in quadruplicates and luciferase readings were taken as 
singlets. Ratios of firefly to Renilla luciferase readings were taken for each experiment and normalized to the activity of the control construct to produce fold differences in luminescence. For further detailed information see Supplementary Materials/Methods.

In silico and in vitro analyses of DNA-protein interactions. An in silico analysis of the putative transcription factors (TFs) binding to the rs105431668 region was performed using ConSite, ${ }^{37}$ which revealed a putative cyclic adenosyl monophospate responsive element-binding protein (CREB)-binding site. Electrophoretic mobility shift assay (EMSA) was then used to investigate in vitro DNA-protein interactions at the rs 105431668 locus. EMSA was performed using the LightShift Chemiluminescent EMSA kit according to the manufacturer's protocol (Thermo Fisher Scientific Inc. Rockford, IL, USA). Competition reactions were performed with 200-fold molar excess of unlabeled ('cold') DNA probes. Antibody (supershift) reactions were performed with the addition of $2 \mu$ lanti-CREB1 (ab5803; Abcam plc, Cambridge, England). The probes used were: FRL probe (the T-allele of rs105431668 is underlined); 5'-ATAAAAGCCCGTTGGTG ACCCGCTCTACGCAT-3', FSL probe (the C-allele of rs105431668 is underlined); 5'-ATAAAAGCCCGTTGGCG ACCCGCTCTACGCAT-3'. Note that the FRL/FSL probes are identical except for the SNP locus.

In vivo chromatin immunoprecipitation. To investigate in vivo DNA-protein interactions and histone modifications (HMs), chromatin immunoprecipitation (ChIP) experiments were performed according to Martens et al. ${ }^{38}$ for a number of candidate TFs and coactivators (TF ChIP), and for HMs (HM ChIP). The antibodies used for TF ChIPs were the following: anti-CREB1 (1:50 dilution; \#9197, Cell Signaling Technology Inc., Danvers, MA, USA), anti-CREB2 (also designated activating TF 4 (ATF4), 1:200; sc-200, Santa Cruz Biotechnology Inc., Santa Cruz, CA, USA), anti-ATF2 (1:200; sc187, SantaCruz), anti-E1A-binding protein p300 (Ep300, 1:200; sc-584, SantaCruz), anti-acetyl-Ep300/CREB-binding protein (acetylp300/CREBBP aka CBP, 1:25; \#4771, Cell Signaling), anti-p300/CBP-associated factor (PCAF, 1:25; \#3378, Cell Signaling), and anti-immunoglobulin G (IgG, $2 \mu \mathrm{l}$; \#2729, Cell Signaling) as negative control. The reactions for HM ChIPs included: anti-histone 3 lysine 18 acetylation

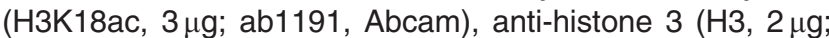
ab1791, Abcam), and anti-histone 3 lysine 27 tri-methylation (H3K27me3, $4 \mu \mathrm{g}$; ab6002, Abcam) and no antibody [Ab( - )] as negative controls. The TF ChIPs were performed both for a 'target' region (that is, containing the rs105431668 locus) and a 'negative' region (that is, upstream from and not containing the rs105431668 locus) in FRL and FSL $(n=6 /$ group; outliers: $n=2$ FRL for CREB2, ATF2 and Ep300). The HM ChIPs were performed for a region close to Npy's TSS in FRL and FSL ( $n=6$ /group for H3K18ac, H3K27me3 and $\mathrm{Ab}(-) ; n=3$ /group for H3). HM ChIPs were also performed following wheel running experiments (described in the next section) in FSL and FSL-runners (H3K18ac and $\mathrm{Ab}(-): n=6$ FSL and $n=5$ FSL-runners, H3: $n=4 \mathrm{FSL}$ and $n=4 \mathrm{FSL}-$ runners; outlier: $n=1 \mathrm{FSL}$ ). For further detailed information see Supplementary Materials/Methods.
Wheel running experiments. To investigate putative epigenetic effects of physical activity on Npy, wheel running experiments were performed as previously described. ${ }^{32}$ In brief, FSL animals were individually housed with either free access or no access to running wheels during a period of 5 weeks. Running data were sampled 48 times per day using a computer-based data system with customized software. Following wheel running experiments, hippocampi were dissected and mRNA levels of candidate genes were measured using qRT-PCR as described previously.

Statistical analyses. Data are presented as mean values and error-bars represent \pm 1 s.e.m. Mann-Whitney U-tests were used for group comparisons. Statistical significance was set at $P<0.05$. All analyses were performed using IBM SPSS Statistics (IBM Corporation, Armonk, NY, USA). Samples with values showing striking (arbitrary) difference from the rest of the group were considered outliers and were excluded from the statistical analyses.

\section{Results}

Genotypic and gene expression differences of Npy between FSL and FRL. DNA sequencing of the upstream promoter and the coding regions of Npy showed that the FSL differed genetically from the $\mathrm{FRL}$ only in the promoter region. More specifically, we found three genetic variations in the proximal promoter of Npy. When cross-referencing with NCBl's SNP database (Entrez dbSNP), all three nt substitutions were found to be registered as rat (Rattus norvegicus) SNPs. The reference SNP (rs) numbers, as reported in genomic build 136, were the following: (1) rs8153612 (A/G), (2) rs107377172 (A/C), (3) rs105431668 (C/T); numbered $5^{\prime}$ to $3^{\prime}$ (Figure 1). Subsequent SNP genotyping studies revealed the presence of two distinct haplotypes in the two strains. More specifically, all of the FRL were homozygous for the $\mathrm{G}-\mathrm{A}-\mathrm{T}$ haplotype, whereas $70 \%$ of the FSL were homozygous for the $A-C-C$ haplotype and $30 \%$ of the FSL were heterozygous for the two haplotypes. Gene expression analyses using qRT-PCR and whole hippocampal homogenates showed that the Npy mRNA levels were decreased in FSL compared with FRL $(P<0.05$; Figure 2a). In situ hybridization experiments using a separate group of animals confirmed the Npy decrease in FSL and showed that it was more pronounced in the CA1 hippocampal region $(P<0.01$; Figure $2 b)$. A similar trend was also present in the dentate gyrus $(P=0.05)$ and in the CA3 $(P=0.11)$ regions of the hippocampus (Figure $2 \mathrm{~b}$ ).

In vitro functionality of the rs105431668 (C/T) SNP. Two of the identified SNPs were located close to conserved Npy promoter elements. Specifically, the second SNP (rs107377172; A/C) was located - 3 nt from Npy's GC-box and the third SNP (rs105431668; C/T) was located + $10 \mathrm{nt}$ from the TATA-box and - $15 \mathrm{nt}$ from the TSS (Figure 1). In order to examine putative SNP functionality, we used a luciferase reporter assay and luminescence data were verified in two different cell lines (HCT-116 and U2OS). Figure $3 a$ indicates the names of the cloned fragments and 
the different SNP haplotypes contained in these clones. Data were first generated using constructs containing either upstream promoter clones (that is, clones with the complete FRL or FSL haplotype) or core promoter clones (that is, clones containing only rs105431668; the SNP closest to the TATA-box). The clones containing the FRL genotype had a positive effect on gene expression, both in the upstream form $(P<0.05$; Figure $3 \mathrm{~b})$ and in the core ( $\mathrm{T}$-allele) promoter form $(P<0.05$; Figure 3c). Results from the core clones suggested that rs105431668 was the functional SNP. However, we

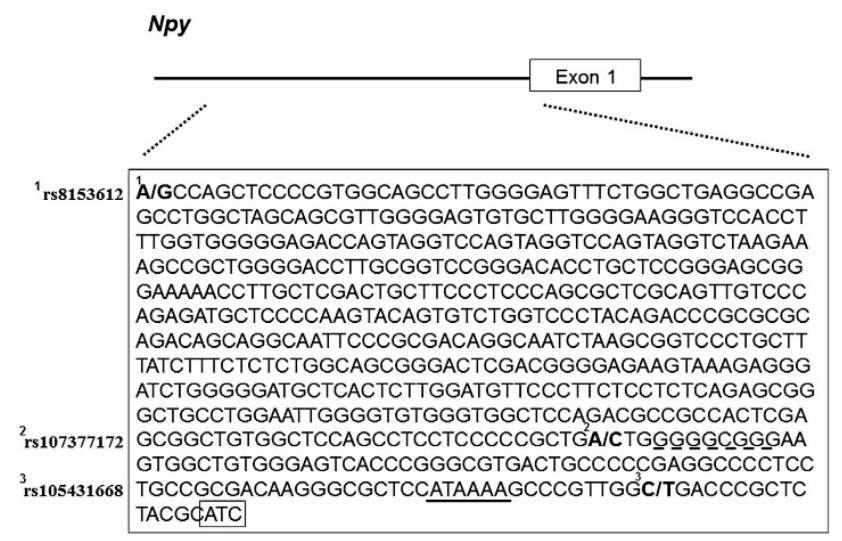

Figure 1 The sequence of the rat's neuropeptide $Y(N p y)$ proximal promoter and transcription start site (TSS) $(-605$ to +3 nt; NCBI Reference Sequence: NC_005103.3 from base 144233162 to 144233769) shown in relation to exon 1 of the Npy gene. The three identified single-nucleotide polymorphisms (SNPs) are printed in bold and their rs-IDs are denoted to the left (1: rs8153612 (A/G), 2: rs107377172 (A/C), 3: rs105431668 (C/T)). Genotyping analyses showed that the Flinders resistant line (FRL) haplotype was $\mathrm{G}-\mathrm{A}-\mathrm{T}$, whereas the different Flinders sensitive line (FSL) haplotype was A-C-C. The conserved GC-box is brokenunderlined, the conserved TATA-box is solid-underlined and the TSS is shown in a box. could not exclude the possible involvement of the two remaining SNPS (rs8153612 and rs107377172) in gene expression regulation. An FRL clone with a chimeric haplotype (that is, $\mathrm{A}-\mathrm{C}-\mathrm{T}$; Figure $3 \mathrm{a}$ ) was able to resolve this issue. This clone contained the first two upstream FSL SNPs and the third (putatively functional) core FRL SNP. By comparing the FSL-upstream/FRL-core clone's activity with the upstream FSL clone, we were able to confirm that the T-allele of rs105431668 (present in all FRL rats) determined the in vitro haplotype-driven transcriptional upregulation $(P<0.05$; Figure $3 d)$. EMSA was then performed to study putative in vitro DNA-protein interactions at the rs105431668-containing region. Significantly, we observed a strong mobility shift band only when using an FRL probe (containing the T-allele of rs105431668; Figure 3e, lanes 2 and 4) but not when using an FSL probe (containing the C-allele of rs105431668; Figure 3e, lanes 6 and 8). The in silico analysis, which was performed to predict putative TFs binding to the rs105431668-containing region, indicated a non-consensus cyclic adenosyl monophospate response element-binding site only under the presence of the T-allele. There are several cyclic adenosyl monophospate response element-binding proteins within the ATF/CREB family, including CREB1, CREB2 (also designated ATF4) and ATF2. Before proceeding with in vivo analyses of these TFs, we also performed EMSA with an anti-CREB1 antibody. However, the latter experiment argued against an in vitro binding of CREB1, as no supershift was observed (Figure 3e; lane 4). In summary, these data suggest a functional DNAprotein interaction within Npy's promoter region under the presence of rs105431668's T-allele.

In vivo allele-specific epigenetic states of Npy. To study putative in vivo protein-DNA interactions, we performed ChIP experiments for three members of the ATF/CREB a

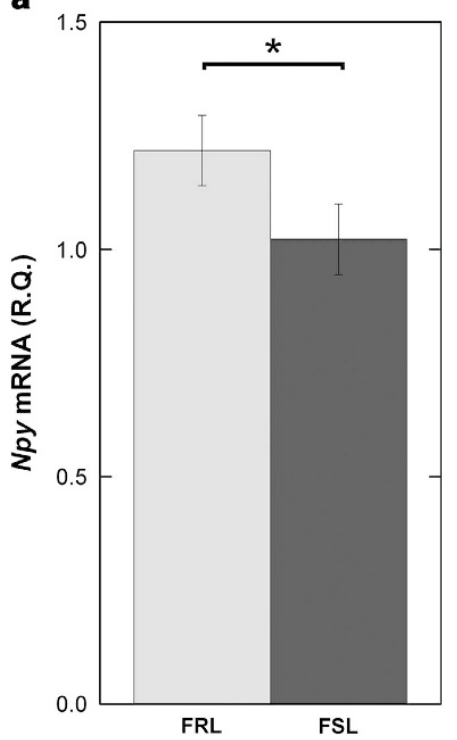

b

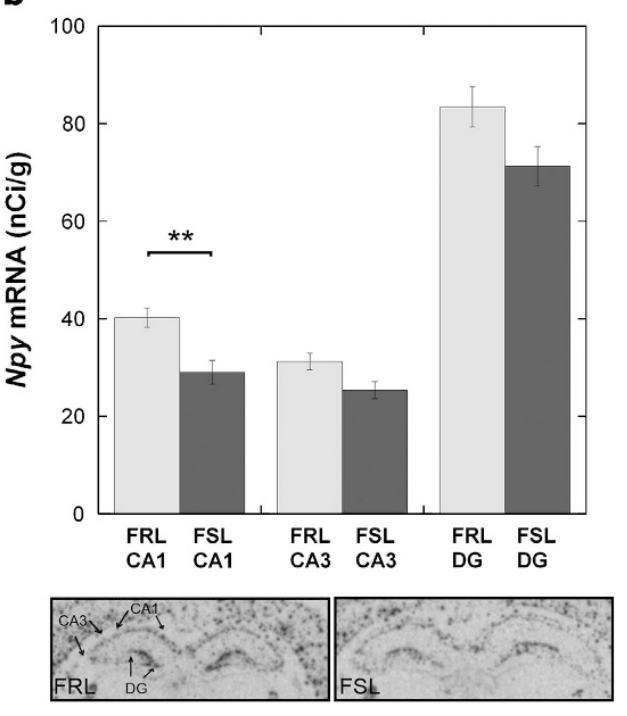

Figure 2 Gene expression analyses of Npy in (a) whole hippocampal homogenate using quantitative real-time PCR (qRT-PCR) and (b) different hippocampal regions using in situ hybridization (representative in situ autoradiograms are shown below the figure). Analyses show a decrease in neuropeptide $Y$ (Npy) mRNA levels, in the hippocampus of the Flinders sensitive line (FSL), which is most pronounced in the cornu ammonis (CA) 1 area. ${ }^{*} P<0.05,{ }^{* *} P<0.01$. 


\begin{tabular}{|l|l|l|}
\hline Name of cloned fragment & Figure abbreviation & SNP haplotype (1-2-3) \\
\hline FRL upstream promoter & FRL-up & G-A-T \\
FSL upstream promoter & FSL-up & A-C-C \\
FRL core promoter & FRL-core & - \\
FSL core promoter & FSL-core & $-\mathrm{T}$ \\
FSL upstream with FRL core & FSL-up/FRL-core & A-C-T \\
\hline
\end{tabular}
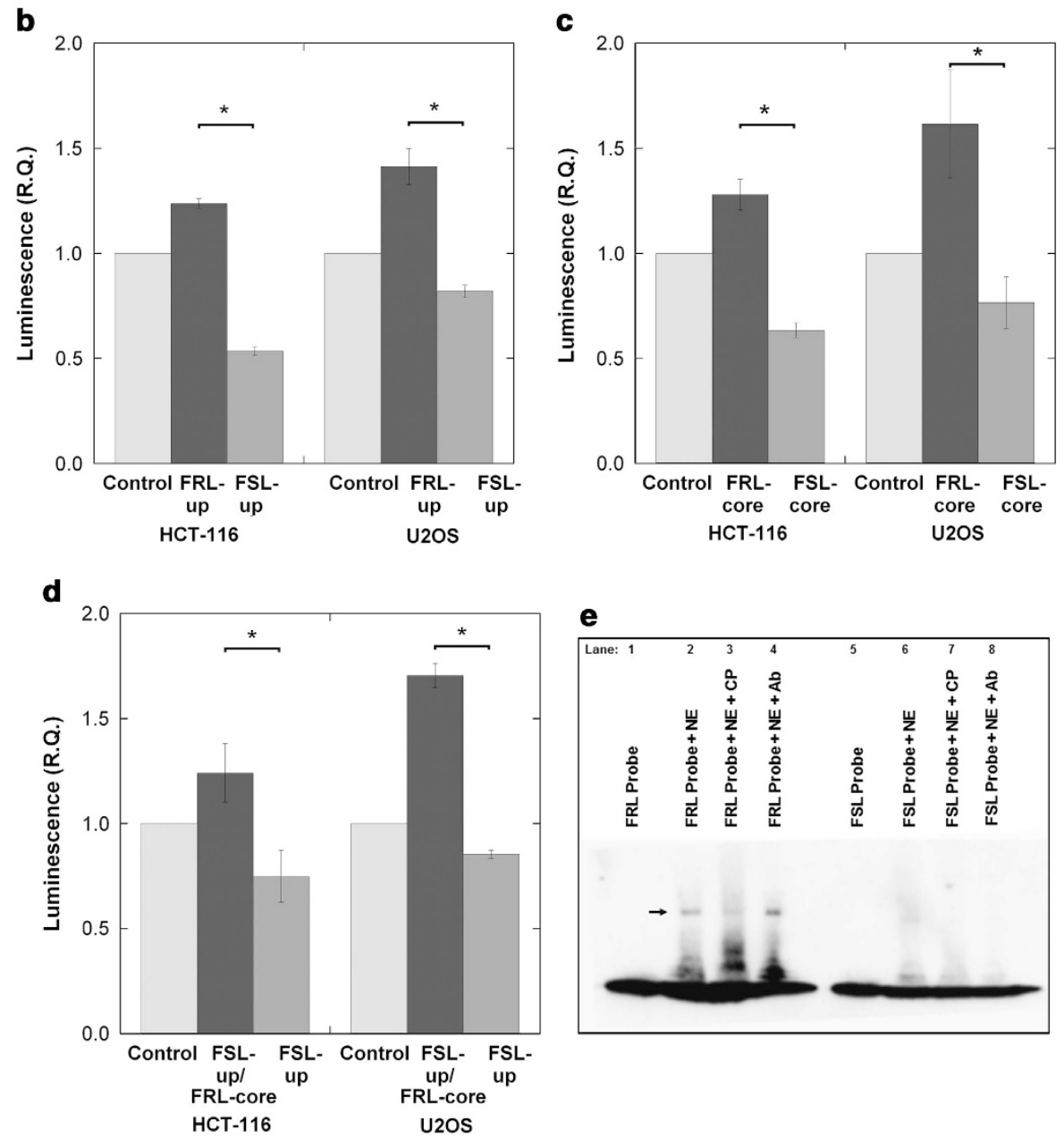

Figure 3 Luciferase reporter assays and electrophoretic mobility shift assay (EMSAs) were performed to investigate the in vitro transcriptional functionality of the three single-nucleotide polymorphism (SNPs) located in the neuropeptide Y (Npy) promoter region of Flinders resistant line (FRL)/Flinders sensitive line (FSL) rats and the putative DNA-protein interactions at the rs 105431668 locus, respectively. (a) Luciferase reporter constructs included either the upstream Npy promoter region (containing all three SNPs: $5^{\prime}$ - rs8153612 - rs107377172-rs105431668-3' in a wild-type or a chimeric (FSL upstream with FRL core) form) or the core promoter region (containing only rs105431668). (b, c, d) Collectively, by using two different cell lines (HCT-116 and U2OS), the different luciferase constructs demonstrated that the rs105431668's T-allele (present in homozygosity only in the FRL rats) leads to increased transcription in vitro compared with the C-allele (present only in the FSL rats). (e) Lanes one and five of the mobility shift assays mark the positions of the free (non-interacting) FRL and FSL probes, respectively. Lane two demonstrates an in vitro DNA-protein interaction after the addition of nuclear extract, which is shown as a gel-shift next to the arrow. A strong shift was only generated by the FRL probe (containing the T-allele of rs105431668; lane 2) and not by the FSL (C-allele) probe (lane 6). Lane three shows the reduction of the DNA-protein interaction that occurs after the addition of 'cold' (unlabeled) probe and indicates specificity in the type of DNA-protein interaction between FRL probe and components of the nuclear extract. Lanes 4 and 8 test for putative supershifts by the addition of an antibody (Ab) against CREB1. No supershifts were observed indicating that CREB1 isn't responsible for the in vitro DNA-protein interaction. Convincingly, however, the initial gel shift is reestablished at the same position for FRL (lane 4) and, again, no shift is observed for FSL (lane 8). ${ }^{*} P<0.05$.

family (CREB1, CREB2 and ATF2) using hippocampal tissue from FSL/FRL animals. As members of the ATF/ CREB family are known to recruit transcriptional coactivators with histone modifying properties, we also examined interactions with Ep300 and PCAF using the same technique. ChIP was performed both for a target region (the region containing rs105431668) and an upstream negative region (not containing rs105431668). The ChIP data revealed an enrichment of CREB2 and Ep300 in the target region of the FRL, but not that of the FSL $(P<0.05$; Figure 4a). There was also an enrichment of Ep300 when using a second antibody against its more active (acetylated) form $(P<0.05$; Figure $4 \mathrm{a})$. None of these enrichments were present in the negative region, suggesting specificity to the rs105431668 locus (Supplementary Figure S1a). In line with these data, and as Ep300 is known to acetylate lysine 18 of histone 3 
a

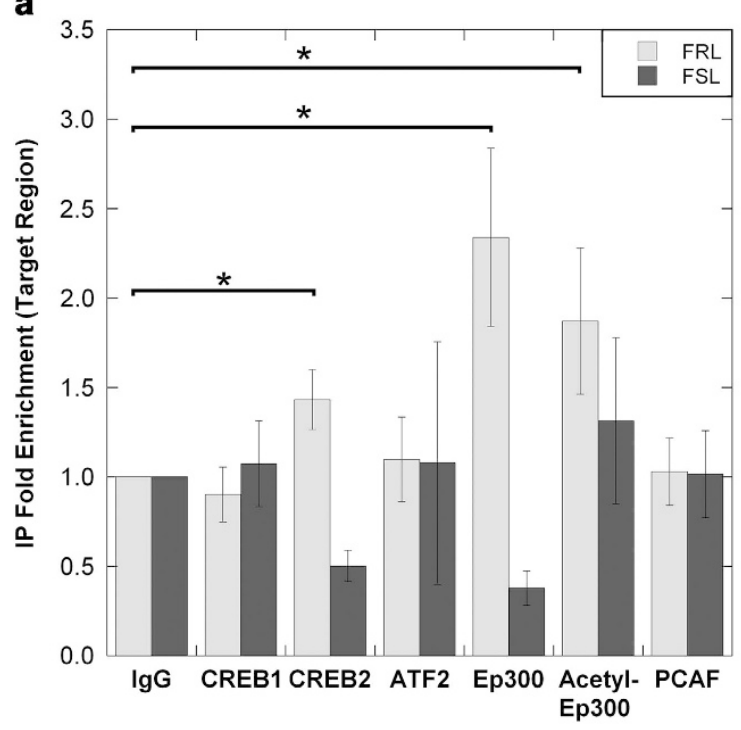

b

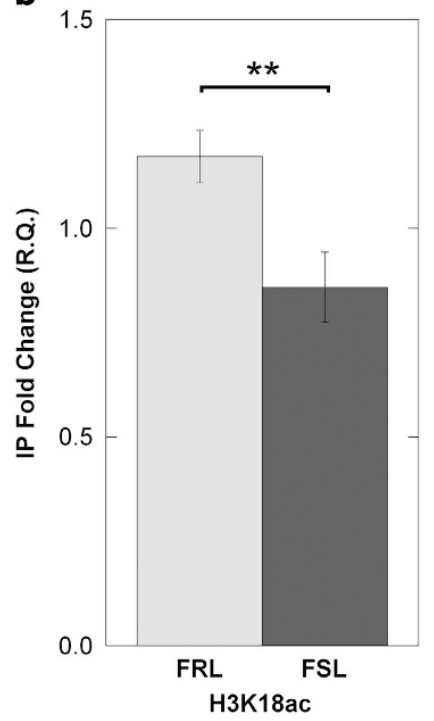

Figure 4 Chromatin immunoprecipitation (ChIP) experiments were performed for transcription factors (TF ChIP) and histone modifications (HM ChIP) in Flinders resistant line (FRL) and Flinders sensitive line (FSL). (a) TF ChIP at the rs105431668-containing (target) region showed an enrichment of CREB2, Ep300, and acetylated Ep300 only in the FRL. (b) As Ep300 is one of the main histone acetyltransferases (HATs) necessary for in vivo maintenance of H3K18ac, we performed HM ChIP against H3K18ac of Npy. In line with Ep300's HAT activity, the HM ChIP showed increased levels of H3K18ac in FRL compared with FSL. ${ }^{*} P<0.05,{ }^{*} P<0.01$.

(H3K18ac), subsequent ChIP experiments showed increased levels of H3K18ac at the Npy region of FRL compared with FSL $(P<0.01$; Figure $4 \mathrm{~b})$. No such difference was found for an Ep300-unrelated HM (H3K27me3) or for levels of H3 (Supplementary Figure S1b). Collectively, these results suggest that dysregulation of $\mathrm{Npy}$ in the hippocampus of FSL is a result of epigenetic modifications that are associated with the rs105431668 genotype. Specifically, the FRL's SNP genotype is associated with recruitment of both CREB2 and the acetylated form of Ep300. Ep300, in turn, leads to a transcriptionally active chromatin state; indicated by the increased levels of both H3K18ac and Npy mRNA in the FRL.

Physical activity is associated with an epigenetic reprogramming of $\mathbf{N p y}$. Physical activity in the form of wheel running was associated with an increase in hippocampal Npy mRNA levels in FSL-runners, compared with FSL rats with no access to running wheels $(P<0.05$; Figure $5 \mathrm{a})$. In accord with the histone-modification findings using naive FSL/FRL rats, the upregulation of Npy's mRNA in FSL-runners was associated with increased levels of H3K18ac $(P<0.05$; Figure 5b). Again, there was no difference in levels of H3 (Supplementary Figure S2a). As an exploratory analysis of the underlying mechanism leading to increased acetylation levels of $\mathrm{H} 3 \mathrm{~K} 18$, we measured the mRNA levels of Ep300 and all known nuclear HDACs. Notably, we found that there was a downregulation of Hdac5 mRNA levels in the running group $(P<0.01$; Figure $5 \mathrm{c})$. No differences were found in the mRNA levels of Ep300 (Supplementary Figure S2b) or all other tested HDACs (Supplementary Figure S2c). These data suggest that physical activity can lead to an activating epigenetic reprogramming of $\mathrm{Npy}$ through the downregulation of Hdac5, which potentially drives the increase of H3K18ac and is not dependent on the genetic variation present at the rs105431668 SNP locus.

\section{Discussion}

General. NPY is suggested to confer resilience to both depression and anxiety; two disorders that are influenced by common genetic factors. ${ }^{39-41}$ This is supported by evidence showing that NPY is reduced in the cerebrospinal fluid of depressed subjects ${ }^{2,42}$ and in post-mortem brains of suicide victims. ${ }^{43}$ In addition, polymorphisms in the human NPY gene have been associated with depression ${ }^{1,2,4,5}$ and a genetic variation in the NPY promoter (SNP rs16147) has been shown to control NPYs transcriptional activity and to affect emotional processing and stress response. ${ }^{3,17,18}$ Studies in the FSL genetic model of depression, have also consistently been in line with NPY's role in the pathophysiology of the disorder. ${ }^{23,24,27,35,36,44-48}$ The present investigation was the first to hypothesize and demonstrate that the observed dysregulation of $\mathrm{Npy}$ in the FSL is a result of a functional SNP variation (rs105431668) resembling human rs16147. It should, however, be noted that, on the level of the individual SNPs, a cross-species comparison is not possible as the human rs16147 and the rat rs105431668 are not orthologous (the rat SNP is located between the Npy's TATAbox and the TSS, whereas the human SNP is located upstream of the TATA-box) and, thus, extrapolation of the presented rat data into humans should be done with caution.

SNP-driven Npy expression and binding of CREB2. Sequence variants that alter gene expression by $\geqslant 1.5$-fold have been found to predominantly localize in the core and proximal promoter gene regions. ${ }^{49}$ Accordingly, using the FSL/FRL model we found that a SNP in the core promoter 
a

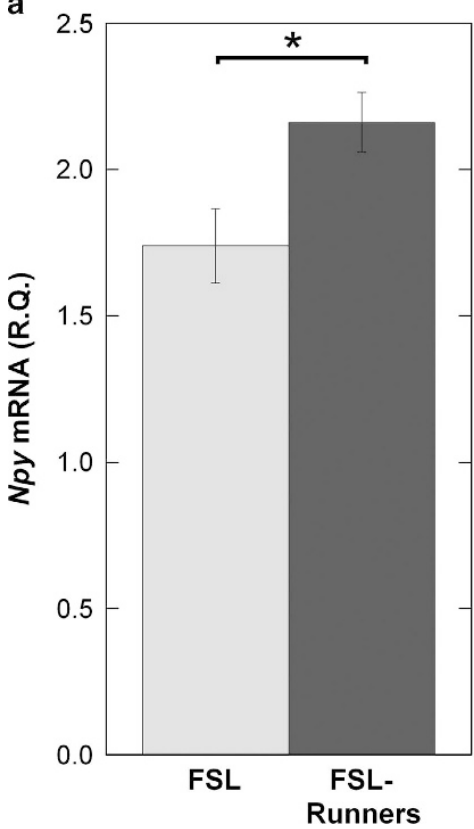

b

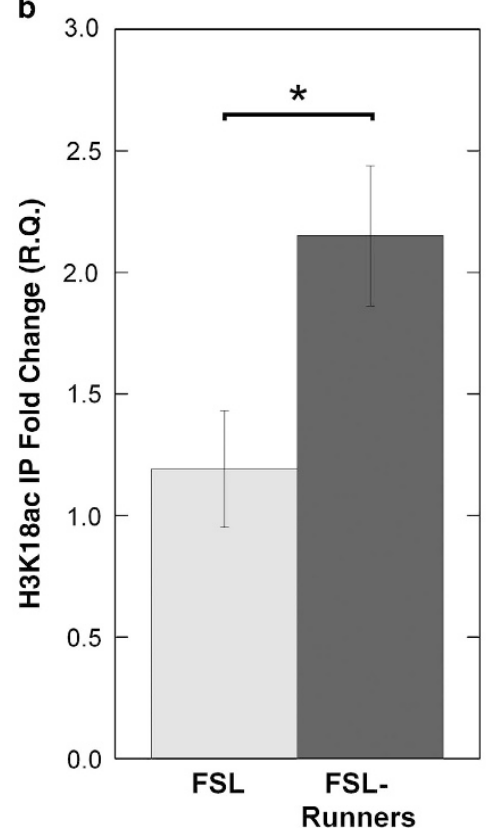

C

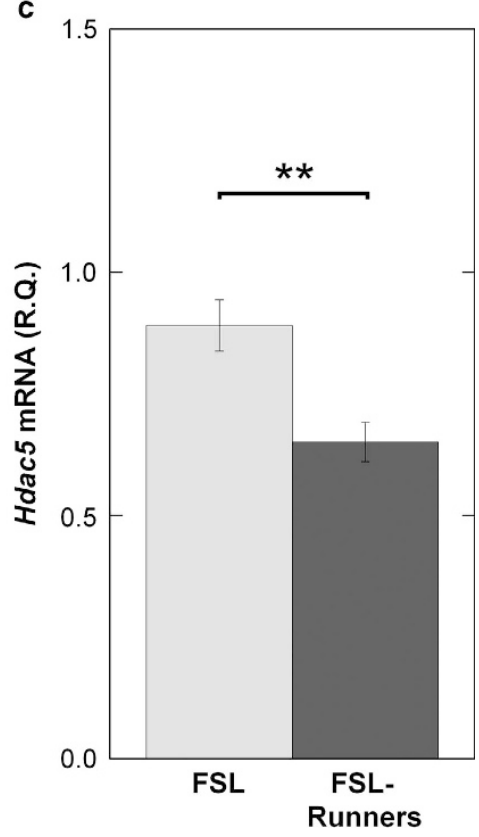

Figure 5 Epigenetic analyses of Neuropeptide (Npy) in hippocampus following wheel running in Flinders sensitive line (FSL)-runners vs FSL with no access to running wheel. Running was associated with (a) an increase in Npy mRNA levels, (b) an increase in levels of H3K18ac and (c) a decrease in mRNA levels of Hdac5. ${ }^{*} P<0.05$, ${ }^{* *} P<0.01$

region of the rat $\mathrm{Npy}(\mathrm{C} / \mathrm{T} ; \mathrm{rs} 105431668)$ was associated with a transcriptional programming. In particular, the FSL rats harbored the C-allele of rs105431668, which was associated with downregulated in vitro transcriptional activity. In addition, in vivo experiments revealed reduced Npy mRNA levels in the hippocampus of FSL, especially in the CA1 area; in accord with previous in situ experiments in the FSL model by Caberlotto et al. ${ }^{23,45}$ In line with a functional role of rs105431668, in vitro assays showed that the SNP's T-allele (present in homozygosity only in the FRL) accounts for a DNA-protein interaction. Subsequent in vivo experiments demonstrated an enrichment of the TF CREB2 (ATF4), only in the Npy promoter region of the FRL containing the T-allele of rs105431668. Cytosolic CREB2 is known to interact with metabotropic gamma-aminobutyric acid $B$ receptors $\left(G A B A_{B} R\right)$ and, upon receptor activation, CREB2 relocates to the nucleus where it exerts its transcriptional regulatory functions. $^{50}$ GABA $_{B} R$ and NPY coexist at GABAergic terminals of hippocampal interneurons, ${ }^{51,52}$ supporting a $\mathrm{GABA}_{B} \mathrm{R}-\mathrm{NPY}$ regulatory pathway that is mediated by CREB2. As CREB2 is a known suppressor of long-term synaptic plasticity and memory, ${ }^{53}$ extrapolating these data may indicate that NPY is also a downstream regulator involved in memory suppression. This hypothesis is consistent with NPY's inhibitory synaptic effects that involve inhibition of adenylyl cyclase (thus also of the production of cyclic adenosyl monophospate) through its binding to postsynaptic NPY-Y1 receptors. ${ }^{51}$ Therefore, NPY's role in mental resilience may be to dampen the molecular effects of adverse experiences. These effects could involve long-term changes in synaptic strengths that get associated with negative memories. Such an assumption would be in line with findings in humans showing that the effect of rs16147 is modulated by early-life adversities ${ }^{5,19-21}$ and also with animal experiments showing that the FSL, but not the FRL, exhibit anhedonic responses only after chronic mild stress. ${ }^{54}$

Allele-specific binding of Ep300 and corresponding HMs. Histone acetyltransferases Ep300 and CBP can be recruited to promoter regions by interacting with $C R E B$ proteins. ${ }^{55-58} \mathrm{PCAF}$, which binds to Ep300/CBP protein complexes, has also an intrinsic histone acetyltransferase activity. ${ }^{59}$ In the present study, in vivo experiments supported an Ep300-binding to CREB2. More specifically, Ep300 was found to be enriched in its acetylated form, which has a higher catalytic activity, ${ }^{60}$ solely in the FRL's Npy promoter region containing the T-allele of rs105431668. CREB2 interaction with Ep300 is known to induce CREB2 stabilization by inhibiting its ubiquitination. ${ }^{61}$ As Ep300 is one of the main histone acetyltransferases necessary for in vivo maintenance of global H3K18ac, ${ }^{62}$ we also performed in vivo experiments to analyze HMs at the Npy locus. Accordingly, we found that the FRL had elevated hippocampal levels of H3K18ac. Owing to lack of available antibody, we were not able to specifically screen for CBP; however, the absence of PCAF enrichment (that binds to the Ep300/CBP complex) provides indirect evidence against the presence of CBP at the Npy promoter region. $\mathrm{H} 3 \mathrm{~K} 18 \mathrm{ac}$ is a $\mathrm{HM}$ that is associated with increased gene activity ${ }^{63}$ and may account for the observed upregulated levels of Npy mRNA in the hippocampus of the FRL. Notably, CREB2 and Ep300 have also been shown to regulate cyclooxygenase-2 $(\mathrm{COX}-2) .{ }^{64} \mathrm{COX}-2$ is expressed throughout the nervous system and is involved in inflammation. ${ }^{65}$ Signs of inflammatory processes resulting 
from the function of COX-2 (for example, increased proinflammatory cytokines) have repeatedly been described in depression and COX-2 has been used as a target in pilot antidepressant studies. ${ }^{66}$ Thus, the comorbidity of depression and inflammation may partly involve the action of CREB2, which relocates from the cytoplasm to the nucleus upon $\mathrm{GABA}_{B} \mathrm{R}$ activation ${ }^{50}$ via, for example, a stressful event and in order to produce neuroprotective effects. This may also explain, for instance, why seizures have been found to stimulate both NPY and COX-2 expression in the hippocampus. ${ }^{51,67}$

\section{Physical activity and epigenetic reprogramming of $\mathbf{N p y}$.} Experiments using the FSL/FRL model have previously shown that physical activity has antidepressant-like behavioral effects and increases both hippocampal neurogenesis and Npy expression. ${ }^{32,33,36}$ In the present study, we found that FSL-runners had increased levels of hippocampal Npy mRNA and H3K18 acetylation. Notably, this increase was associated with a reduction in the mRNA levels of a specific histone modifying enzyme; Hdac5. HDAC5 is a class II HDAC known to interact with and repress myocyte enhancer factor-2 TFs needed for skeletal myogenesis. ${ }^{68}$ More specifically, physical exercise and increases in motor neuron activity lead to a calcium influx that stimulates the expression of myocyte enhancer factor-2 responsive muscle-specific genes by phosphorylation-dependent inactivation and cytoplasmic translocation of nuclear class II HDACs. ${ }^{68}$ The decreased levels of Hdac5 mRNA in FSL, as a response to physical activity, suggest a transcription-dependent regulation of Hdac5 in the hippocampus. This result is in line with a recent study investigating the expression of the brain-derived neurotrophic factor (Bdnf) gene in the rat hippocampus: physical activity was associated with an increase in $\mathrm{H} 3$ acetylation and Bdnf expression, and a decrease in HDAC5 protein and gene expression. ${ }^{69}$ In addition, imipramine has been shown to increase histone acetylation of Bdnf and also selectively decrease Hdac5 mRNA levels in the mouse hippocampus. ${ }^{70}$ As Bdnf and Npy are both considered resilience-conferring genes, ${ }^{7}$ these data indicate commonalities in their regulatory pathways.

\section{Conclusion}

Our results suggest that the rat rs105431668 is a functional Npy SNP, which may explain depression-related molecular and behavioral characteristics of the FSL model. The presented data also provide clues about the epigenotypic architecture of depression by demonstrating how certain HMs are associated with Npy's transcriptional activity through allele-specific recruitment of certain TFs and coactivators. Additionally, even though genetic at its basis, the disadvantageous epigenetic state of Npy in the FSL was rescued by physical activity. Physical activity has been suggested to have antidepressant effects and recruit processes that confer stress resilience in humans. ${ }^{71,72}$ Physical activity intervention studies, in combination with molecular experiments exploring genes like $N P Y$ and $B D N F$, are thus warranted in clinical settings and could provide insights into novel non-pharmacological and pharmacological antidepressant treatments.

\section{Conflict of interest}

The authors declare no conflict of interest.

Acknowledgements. This work was supported by the Karolinska Institutet's Faculty Funds (KID; PAM), the Bodossaki Foundation (PAM), the Swedish Research Council (research grants: AAM and CL; and postdoctoral fellowship: HVN) and the regional agreement on medical training and clinical research (ALF) between Stockholm County Council and Karolinska Institutet (CL). These funding sources had no involvement in the study design, the collection, analysis and interpretation of the data, in the writing of the manuscript, or in the decision to submit the report for publication.

1. Bosker FJ, Hartman CA, Nolte IM, Prins BP, Terpstra P, Posthuma D et al. Poor replication of candidate genes for major depressive disorder using genome-wide association data. Mol Psychiatry 2011; 16: 516-532.

2. Heilig M, Zachrisson O, Thorsell A, Ehnvall A, Mottagui-Tabar S, Sjogren M et al. Decreased cerebrospinal fluid neuropeptide Y (NPY) in patients with treatment refractory unipolar major depression: preliminary evidence for association with preproNPY gene polymorphism. J Psychiatr Res 2004; 38: 113-121.

3. Mickey BJ, Zhou Z, Heitzeg MM, Heinz E, Hodgkinson CA, Hsu DT et al. Emotion processing, major depression, and functional genetic variation of neuropeptide $Y$. Arch Gen Psychiatry 2011; 68: 158-166.

4. Sjoholm LK, Melas PA, Forsell Y, Lavebratt C. PreproNPY Pro7 protects against depression despite exposure to environmental risk factors. J Affect Disord 2009; 118: 124-130.

5. Sommer WH, Lidstrom J, Sun H, Passer D, Eskay R, Parker SC et al. Human NPY promoter variation rs16147:T $>C$ as a moderator of prefrontal NPY gene expression and negative affect. Hum Mutat 2010; 31: E1594-E1608.

6. Cohen H, Liu T, Kozlovsky N, Kaplan Z, Zohar J, Mathé AA. The neuropeptide Y (NPY)ergic system is associated with behavioral resilience to stress exposure in an animal model of post-traumatic stress disorder. Neuropsychopharmacology 2012; 37: 350-363.

7. Feder A, Nestler EJ, Charney DS. Psychobiology and molecular genetics of resilience. Nat Rev Neurosci 2009; 10: 446-457.

8. Sajdyk TJ, Johnson PL, Leitermann RJ, Fitz SD, Dietrich A, Morin M et al. Neuropeptide Y in the amygdala induces long-term resilience to stress-induced reductions in social responses but not hypothalamic-adrenal-pituitary axis activity or hyperthermia. J Neurosci 2008; 28: 893-903.

9. Thorsell A, Michalkiewicz M, Dumont Y, Quirion R, Caberlotto L, Rimondini R et al. Behavioral insensitivity to restraint stress, absent fear suppression of behavior and impaired spatial learning in transgenic rats with hippocampal neuropeptide $Y$ overexpression. Proc Natl Acad Sci USA 2000; 97: 12852-12857.

10. Bertocchi I, Oberto A, Longo A, Mele P, Sabetta M, Bartolomucci A et al. Regulatory functions of limbic $Y 1$ receptors in body weight and anxiety uncovered by conditional knockout and maternal care. Proc Natl Acad Sci USA 2011; 108: 19395-19400.

11. Decressac M, Wright B, David B, Tyers $P$, Jaber M, Barker RA et al. Exogenous neuropeptide $Y$ promotes in vivo hippocampal neurogenesis. Hippocampus 2011; 21: 233-238.

12. Bremner JD, Narayan M, Anderson ER, Staib LH, Miller HL, Charney DS. Hippocampal volume reduction in major depression. Am J Psychiatry 2000; 157: 115-118.

13. Campbell S, Marriott M, Nahmias C, MacQueen GM. Lower hippocampal volume in patients suffering from depression: a meta-analysis. Am J Psychiatry 2004; 161: 598-607.

14. MacQueen GM, Campbell S, McEwen BS, Macdonald K, Amano S, Joffe RT et al. Course of illness, hippocampal function, and hippocampal volume in major depression. Proc Natl Acad Sci USA 2003; 100: 1387-1392.

15. Neumeister A, Wood S, Bonne O, Nugent AC, Luckenbaugh DA, Young T et al. Reduced hippocampal volume in unmedicated, remitted patients with major depression versus control subjects. Biol Psychiatry 2005; 57: 935-937.

16. Sheline YI, Wang PW, Gado MH, Csernansky JG, Vannier MW. Hippocampal atrophy in recurrent major depression. Proc Natl Acad Sci USA 1996; 93: 3908-3913.

17. Zhou Z, Zhu G, Hariri AR, Enoch MA, Scott D, Sinha R et al. Genetic variation in human NPY expression affects stress response and emotion. Nature 2008; 452: 997-1001.

18. Domschke K, Dannlowski U, Hohoff C, Ohrmann P, Bauer J, Kugel H et al. Neuropeptide Y (NPY) gene: Impact on emotional processing and treatment response in anxious depression. Eur Neuropsychopharmacol 2010; 20: 301-309.

19. Lindell SG, Schwandt ML, Sun H, Sparenborg JD, Bjork K, Kasckow JW et al. Functional NPY variation as a factor in stress resilience and alcohol consumption in rhesus macaques. Arch Gen Psychiatry 2010; 67: 423-431.

20. Witt SH, Buchmann AF, Blomeyer D, Nieratschker V, Treutlein J, Esser G et al. An interaction between a neuropeptide $Y$ gene polymorphism and early adversity modulates endocrine stress responses. Psychoneuroendocrinology 2011; 36: 1010-1020.

21. Donner J, Sipila T, Ripatti S, Kananen L, Chen X, Kendler KS et al. Support for involvement of glutamate decarboxylase 1 and neuropeptide y in anxiety susceptibility. Am J Med Genet B Neuropsychiatr Genet 2012; 159B: 316-327. 
22. Bahar B, Sweeney T. Mapping of the transcription start site (TSS) and identification of SNPs in the bovine neuropeptide Y (NPY) gene. BMC Genet 2008; 9: 91.

23. Caberlotto L, Fuxe K, Overstreet DH, Gerrard $\mathrm{P}$, Hurd $\mathrm{YL}$. Alterations in neuropeptide $Y$ and $Y 1$ receptor $\mathrm{mRNA}$ expression in brains from an animal model of depression: region specific adaptation after fluoxetine treatment. Brain Res Mol Brain Res 1998; 59: 58-65.

24. Jimenez-Vasquez PA, Overstreet DH, Mathe AA. Neuropeptide $Y$ in male and female brains of Flinders Sensitive Line, a rat model of depression. Effects of electroconvulsive stimuli. J Psychiatr Res 2000; 34: 405-412.

25. Husum $\mathrm{H}$, Vasquez PA, Mathé AA. Changed concentrations of tachykinins and neuropeptide $Y$ in brain of a rat model of depression: lithium treatment normalizes tachykinins. Neuropsychopharmacology 2001; 24: 183-191.

26. Wortwein G, Husum $H$, Andersson W, Bolwig TG, Mathé AA. Effects of maternal separation on neuropeptide $Y$ and calcitonin gene-related peptide in "depressed" Flinders Sensitive Line rats: a study of gene-environment interactions. Prog Neuropsychopharmacol Biol Psychiatry 2006; 30: 684-693.

27. Jimenez-Vasquez PA, Diaz-Cabiale Z, Caberlotto L, Bellido I, Overstreet D, Fuxe K et al. Electroconvulsive stimuli selectively affect behavior and neuropeptide Y (NPY) and NPY $Y(1)$ receptor gene expressions in hippocampus and hypothalamus of Flinders Sensitive Line rat model of depression. Eur Neuropsychopharmacol 2007; 17: 298-308.

28. Overstreet DH, Friedman E, Mathé AA, Yadid G. The Flinders Sensitive Line rat: selectively bred putative animal model of depression. Neurosci Biobehav Rev 2005; 29: 739-759.

29. Chen F, Madsen TM, Wegener G, Nyengaard JR. Imipramine treatment increases the number of hippocampal synapses and neurons in a genetic animal model of depression. Hippocampus 2010; 20: 1376-1384.

30. Gomez-Galan M, De Bundel D, Van Eeckhaut A, Smolders I, Lindskog M. Dysfunctional astrocytic regulation of glutamate transmission in a rat model of depression. Mol Psychiatry 2013; 18: 582-594.

31. Eriksson TM, Delagrange $P$, Spedding M, Popoli M, Mathé AA, Ogren SO et al. Emotiona memory impairments in a genetic rat model of depression: involvement of 5-HT/MEK/Arc signaling in restoration. Mol Psychiatry 2012; 17: 173-184

32. Bjornebekk A, Mathé $A A$, Brene $S$. The antidepressant effect of running is associated with increased hippocampal cell proliferation. Int J Neuropsychopharmacol 2005; 8: 357-368.

33. Bjornebekk A, Mathé AA, Brene $S$. The antidepressant effects of running and escitalopram are associated with levels of hippocampal NPY and Y1 receptor but not cell proliferation in a rat model of depression. Hippocampus 2010; 20: 820-828.

34. Drummond AJ, Ashton B, Buxton S, Cheung M, Heled J, Kearse M et al. Geneious 4.8 edn 2010.

35. Melas PA, Mannervik M, Mathé AA, Lavebratt C. Neuropeptide Y: identification of a nove rat $\mathrm{mRNA}$ splice-variant that is downregulated in the hippocampus and the prefrontal cortex of a depression-like model. Peptides 2012; 35: 49-55.

36. Bjornebekk A, Mathé AA, Brene S. Running has differential effects on NPY, opiates, and cell proliferation in an animal model of depression and controls. Neuropsychopharmacology 2006; 31: 256-264.

37. Sandelin A, Wasserman WW, Lenhard B. ConSite: web-based prediction of regulatory elements using cross-species comparison. Nucleic Acids Res 2004; 32, Web Server issue W249-W252.

38. Martens JH, O'Sullivan RJ, Braunschweig U, Opravil S, Radolf M, Steinlein P et al. The profile of repeat-associated histone lysine methylation states in the mouse epigenome. EMBO J 2005; 24: 800-812.

39. Kendler KS, Neale MC, Kessler RC, Heath AC, Eaves LJ. Major depression and generalized anxiety disorder. Same genes, (partly) different environments? Arch Gen Psychiatry 1992; 49: 716-722

40. Morgan CA 3rd, Wang S, Southwick SM, Rasmusson A, Hazlett G, Hauger RL et al. Plasma neuropeptide-Y concentrations in humans exposed to military survival training. Bid Psychiatry 2000; 47: 902-909.

41. Yehuda R, Brand S, Yang RK. Plasma neuropeptide $Y$ concentrations in combat exposed veterans: relationship to trauma exposure, recovery from PTSD, and coping. Biol Psychiatry 2006; 59: 660-663.

42. Widerlov E, Lindstrom LH, Wahlestedt C, Ekman R. Neuropeptide $Y$ and peptide YY as possible cerebrospinal fluid markers for major depression and schizophrenia, respectively. J Psychiatr Res 1988; 22: 69-79.

43. Widdowson PS, Ordway GA, Halaris AE. Reduced neuropeptide $Y$ concentrations in suicide brain. J Neurochem 1992; 59: 73-80.

44. Mathé AA, Jimenez PA, Theodorsson E, Stenfors $C$. Neuropeptide $Y$, neurokinin A and neurotensin in brain regions of Fawn Hooded "depressed", Wistar, and Sprague Dawley rats. Effects of electroconvulsive stimuli. Prog Neuropsychopharmacol Biol Psychiatry 1998; 22: 529-546.

45. Caberlotto L, Jimenez P, Overstreet DH, Hurd YL, Mathé AA, Fuxe K. Alterations in neuropeptide $Y$ levels and $Y 1$ binding sites in the Flinders Sensitive Line rats, a genetic animal model of depression. Neurosci Lett 1999; 265: 191-194.

46. Stogner KA, Holmes PV. Neuropeptide-Y exerts antidepressant-like effects in the forced swim test in rats. Eur J Pharmacol 2000; 387: R9-10.
47. Jimenez Vasquez PA, Salmi $P$, Ahlenius $S$, Mathé AA. Neuropeptide $Y$ in brains of the Flinders Sensitive Line rat, a model of depression. Effects of electroconvulsive stimuli and d-amphetamine on peptide concentrations and locomotion. Behav Brain Res 2000; 111: 115-123.

48. Jimenez-Vasquez PA, Mathé AA, Thomas JD, Riley EP, Ehlers CL. Early maternal separation alters neuropeptide $Y$ concentrations in selected brain regions in adult rats. Brain Res Dev Brain Res 2001; 131: 149-152.

49. Buckland PR, Hoogendoorn B, Coleman SL, Guy CA, Smith SK, O'Donovan MC. Strong bias in the location of functional promoter polymorphisms. Hum Mutat 2005; 26: 214-223.

50. White JH, Mclllhinney RA, Wise A, Ciruela F, Chan WY, Emson PC et al. The GABAB receptor interacts directly with the related transcription factors CREB2 and ATFx. Proc Natl Acad Sci USA 2000; 97: 13967-13972.

51. Benarroch EE. Neuropeptide Y: its multiple effects in the CNS and potential clinical significance. Neurology 2009; 72: 1016-1020.

52. Ulrich $\mathrm{D}$, Bettler $\mathrm{B}$. GABA(B) receptors: synaptic functions and mechanisms of diversity. Curr Opin Neurobiol 2007; 17: 298-303.

53. Chen A, Muzzio IA, Malleret G, Bartsch D, Verbitsky M, Pavlidis P et al. Inducible enhancement of memory storage and synaptic plasticity in transgenic mice expressing an inhibitor of ATF4 (CREB-2) and C/EBP proteins. Neuron 2003; 39: 655-669.

54. Pucilowski O, Overstreet DH, Rezvani AH, Janowsky DS. Chronic mild stress-induced anhedonia: greater effect in a genetic rat model of depression. Physiol Behav 1993; 54: $1215-1220$.

55. Chrivia JC, Kwok RP, Lamb N, Hagiwara M, Montminy MR, Goodman RH. Phosphorylated CREB binds specifically to the nuclear protein CBP. Nature 1993; 365: 855-859.

56. Kwok RP, Lundblad JR, Chrivia JC, Richards JP, Bachinger HP, Brennan RG et al. Nuclear protein CBP is a coactivator for the transcription factor CREB. Nature 1994; 370: 223-226.

57. Lundblad JR, Kwok RP, Laurance ME, Harter ML, Goodman RH. Adenoviral E1Aassociated protein p300 as a functional homologue of the transcriptional co-activator CBP. Nature 1995; 374: 85-88.

58. Arany Z, Newsome D, Oldread E, Livingston DM, Eckner R. A family of transcriptional adaptor proteins targeted by the E1A oncoprotein. Nature 1995; $374: 81-84$.

59. Yang XJ, Ogryzko VV, Nishikawa J, Howard BH, Nakatani Y. A p300/CBP-associated factor that competes with the adenoviral oncoprotein E1A. Nature 1996; 382: 319-324.

60. Thompson PR, Wang D, Wang L, Fulco M, Pediconi N, Zhang D et al. Regulation of the p300 HAT domain via a novel activation loop. Nat Struct Mol Biol 2004; 11: 308-315.

61. Lassot I, Estrabaud E, Emiliani S, Benkirane M, Benarous R, Margottin-Goguet F. p300 modulates ATF4 stability and transcriptional activity independently of its acetyltransferase domain. J Biol Chem 2005; 280: 41537-41545.

62. Horwitz GA, Zhang K, McBrian MA, Grunstein M, Kurdistani SK, Berk AJ. Adenovirus small e1a alters global patterns of histone modification. Science 2008; 321: 1084-1085.

63. Jenuwein T, Allis CD. Translating the histone code. Science 2001; 293: 1074-1080.

64. Schroer K, Zhu Y, Saunders MA, Deng WG, Xu XM, Meyer-Kirchrath J et al. Obligatory role of cyclic adenosine monophosphate response element in cyclooxygenase-2 promoter induction and feedback regulation by inflammatory mediators. Circulation 2002; 105: 2760-2765

65. Bazan NG. COX-2 as a multifunctional neuronal modulator. Nat Med 2001; 7: 414-415

66. Muller N, Schwarz MJ, Dehning S, Douhe A, Cerovecki A, Goldstein-Muller B et al. The cyclooxygenase-2 inhibitor celecoxib has therapeutic effects in major depression: results of a double-blind, randomized, placebo controlled, add-on pilot study to reboxetine. Mol Psychiatry 2006; 11: 680-684.

67. Marcheselli VL, Bazan NG. Sustained induction of prostaglandin endoperoxide synthase-2 by seizures in hippocampus. Inhibition by a platelet-activating factor antagonist. J Bio Chem 1996; 271: 24794-24799.

68. McKinsey TA, Zhang CL, Lu J, Olson EN. Signal-dependent nuclear export of a histone deacetylase regulates muscle differentiation. Nature 2000; 408: 106-111.

69. Gomez-Pinilla F, Zhuang Y, Feng J, Ying Z, Fan G. Exercise impacts brain-derived neurotrophic factor plasticity by engaging mechanisms of epigenetic regulation. Eur $J$ Neurosci 2011; 33: 383-390.

70. Tsankova NM, Berton O, Renthal W, Kumar A, Neve RL, Nestler EJ. Sustained hippocampal chromatin regulation in a mouse model of depression and antidepressant action. Nat Neurosci 2006; 9: 519-525.

71. Mead GE, Morley W, Campbell P, Greig CA, McMurdo M, Lawlor DA. Exercise for depression. Cochrane Database Syst Rev 2009 CD004366.

72. Salmon P. Effects of physical exercise on anxiety, depression, and sensitivity to stress: a unifying theory. Clin Psychol Rev 2001; 21: 33-61.

Translational Psychiatry is an open-access journal published by Nature Publishing Group. This work is licensed under a Creative Commons Attribution-NonCommercialShareAlike 3.0 Unported License. To view a copy of this license, visit http://creativecommons.org/licenses/by-nc-sa/3.0/ 